\title{
Reflexiones para avanzar: experiencias en la investigación educativa
} Reflections to improve: experiences in educational research

\author{
Patricia Ortega Aguilar, Daniel Traverso-Macías ${ }^{* *}$
}

Recibido: 24 de noviembre de 2020 Aceptado: 17 de febrero de 2021 Publicado: 27 de julio de 2021

To cite this article: Ortega-Aguilar, P. y Traverso-Macias, D. (2021). Reflexiones para avanzar: experiencias en la investigación educativa. Márgenes, Revista de Educación de la Universidad de Málaga. 2 (2), 157-172

DOI: http://dx.doi.org/10.24310/mgnmar.v2i2.10900

\section{RESUMEN}

El siguiente trabajo, de carácter reflexivo, muestra la visión de dos estudiantes de doctorado que, tras iniciarse en el mundo de la investigación educativa, creen necesario compartir sus vivencias y primeros pasos con personas que también estén comenzando su andadura en este ámbito. Así mismo, se pretende compartir con el profesorado encargado de formarlos en este camino, cuáles son las incertidumbres, necesidades y emociones encontradas durante la formación como investigador e investigadora. De este modo, se persigue favorecer una buena formación y fomentar la realización de investigaciones en el contexto educativo con el fin de comprenderlo y ayudar a mejorarlo.

Palabras clave: Investigación educativa; Investigación cualitativa; experiencias investigadoras; formación, autorreflexión

\section{ABSTRACT}

The following reflective work shows the vision of two doctoral thesis students who, after starting in the world of educational research, believe it is necessary to share their experiences and first steps with people who are also beginning their career in this field. Likewise, it is intended to share with the teachers in charge of training them on this path, what are the uncertainties, needs and emotions found during training as a researcher. In this way, the aim is to promote good formation, encourage research in the educational context in order to understand it, and help to improve it.

Keywords: Educative research, Qualitative Research, Research experiences; training, critical reflection

\section{INTRODUCCIÓN}

El ámbito educativo es un terreno lleno de prácticas y metodologías didácticas que, aunque en la actualidad sean reconocidas y llevadas a cabo por muchos y muchas docentes en los procesos de enseñanza-aprendizaje, en algún momento también fueron cuestionadas por la comunidad educativa. Por ello, es necesario un análisis que pueda argumentar si ocasionan beneficios o no dentro de un aula mejorando el aprendizaje de los alumnos y las alumnas. 
Además, resulta imprescindible tener presente que la escuela es un reflejo de la sociedad y viceversa, y que esta última se encuentra en continuo cambio, lo que conlleva una imperante necesidad de cuestionar la realidad de la que formamos parte, intentando mejorarla y ajustarla a las características y necesidades de los niños y las niñas. De ahí que investigar debería ser una práctica continua e irremplazable, ya que permitirá seguir conociendo y profundizando sobre los acontecimientos que tienen lugar en el ámbito educativo. Surge aquí la necesidad de mencionar un concepto que ampara y justifica la postura que se desarrolla a lo largo del artículo, el concepto del profesional-reflexivo. En este sentido, el profesorado debe tener dos habilidades intrínsecas, las docentes y las de investigación, con el fin de poder comprender la realidad en la que ejerce su profesión, así como resolver los problemas y cuestiones que se presenten en el día a día (Domingo, 2020). En palabras de Tallaferro (2006), "se necesita capacitar a los profesores para que se empeñen en procesos cognitivos de pensamiento reflexivo, para que adquieran la capacidad cognitiva y las creencias, valores, actitudes y emociones facilitadoras de tales procesos” (p. 272).

Nosotros ${ }^{1}$, jóvenes nóveles investigadores, maestro de Educación Primaria y maestra de Educación Infantil, sentíamos la investigación como algo desconocido y al alcance de un reducido grupo de personas hasta la realización del máster en Investigación Educativa para el Desarrollo Profesional del Docente (IEDPD), debido a que durante la formación de nuestros respectivos grados, la investigación pasa en buena parte desapercibida, existiendo algunos matices más acentuados en el Grado de Educación Primaria. Es por ello que si hace un par de años alguien nos hubiera preguntado ¿qué es investigar?, probablemente ambos hubiéramos recurrido a la segunda definición que hace la Real Academia de la Lengua de la misma, "realizar actividades intelectuales y experimentales de modo sistemático con el propósito de aumentar los conocimientos sobre una determinada materia” $\left(2019^{2}\right)$. Sin embargo, dicho máster nos abriría la puerta a una nueva dimensión, la dimensión de la investigación educativa. Consideramos que esta línea de investigación va mucho más allá de aumentar los conocimientos sobre una determinada materia, implica intentar transformar las escuelas, siempre mejorándolas y colaborando con estas para ofrecer una educación inclusiva para todos y todas, una educación para el bien común desde la justicia social.

Con el paso del tiempo y el transcurso del máster, no tardamos en reflexionar acerca de qué habíamos hecho todo este tiempo, ¿cómo podía pasar tan desapercibida la investigación durante la formación inicial?, ¿acaso hay mejor fórmula para seguir aprendiendo y formándonos como docentes? Sin duda, poseer herramientas para lograr dar respuestas a todas aquellas preguntas que nos surjan como sujetos críticos en nuestro entorno o con los alumnos y las alumnas, ofrece, en buena parte, la llave maestra para mejorar nuestro contexto y, sobre todo, para satisfacer las necesidades del alumnado consiguiendo así que puedan dar lo mejor de sí mismos y de sí mismas. A pesar de ello, como investigador e investigadora noveles, queda por delante un largo camino de altos y bajos, de ganas y miedos, de certezas e incertidumbres, ya que en este ámbito de la investigación educativa, como en muchos otros, "la práctica hace al maestro y a la maestra" y nos hace tomar conciencia de que solo investigando, aprendes a investigar.

1 En ocasiones, se hará uso de la primera persona del plural con la intención de dotar el discurso de un carácter más personal y cercano.

2 Edición del Tricentenario: https://dle.rae.es/investigar 
Tras nuestro acercamiento a la práctica indagatoria, no concebimos la idea de desempeñar la función docente sin no ser, al mismo tiempo, investigador e investigadora, más cuando el proceso investigación se realiza con mayor frecuencia de lo que se piensa, simplemente cuando se inicia un proceso a través del cual se le quiera dar solución a alguna problemática presente en las aulas. De este modo, desde la presente reflexión, se hace hincapié en la necesidad de trazar un puente entre ambas prácticas (docente e investigadora) para que una se nutra de la otra, en un sentido bidireccional. Por otra parte, también se considera necesario resaltar la idea de que, en muchas ocasiones, la separación existente entre el mundo docente y el investigador hace que las investigaciones llevadas a cabo en estos contextos y sus conclusiones no estén orientadas a que la comunidad educativa pueda ser conocedora de estas y comprendan su valor; a ello se le suma el lenguaje utilizado en los informes finales, y los tecnicismos empleados. En esta línea, en el caso en el que la investigación sea realizada por un agente externo al contexto educativo estudiado, sería indispensable también hacer un feedback con aquellas personas y contextos que hacen posible la investigación, por ser agentes de participación, ya que los resultados pueden ser útiles en la práctica de educativa.

Para concluir este apartado, y de manera previa a narrar nuestras experiencias, se considera oportuno plantear las siguientes cuestiones, ¿pertenece la investigación educativa al profesorado de infantil y primaria?, ¿es algo vinculado al ámbito universitario o toma su verdadero sentido en las aulas y en las realidades educativas?, ¿es necesario hacer hincapié en la importancia del perfil investigador dentro de una de esas características que se añadirían en una posible lista de "buenas prácticas educativas"?

En lo que sigue, y desde estas premisas, haremos una revisión bibliográfica para conocer qué nos cuentan las lecturas a nivel teórico acerca de la investigación y sobre todo en el ámbito educativo para así dar paso a una segunda parte contextualizada sobre nuestras experiencias investigadoras.

\section{QUÉ NOS CUENTAN LAS LECTURAS}

Como se ha nombrado anteriormente, se desarrollarán a nivel genérico los conceptos que se aluden a lo largo de este artículo con la intención de facilitar a un mayor rango de lectores y lectoras la comprensión de nuestras experiencias narradas, generándose así una fotografía del ámbito en el que nos encontramos inmerso e inmersa.

\subsection{Investigación educativa}

En sentido general, cuando preguntamos a cualquier ciudadano o ciudadana qué entiende por investigación o qué características atribuye a esta, la mayoría hace referencia a la investigación arraigada en el contexto científico. Por investigación científica, se entiende que "es un proceso que mediante la aplicación de un método científico nos permite obtener información relevante y fidedigna para entender, verificar, corregir o aplicar el conocimiento" (Tejedor, 2004, p.63). Se podría ir más allá, hablando del positivismo, postura que acoge y enmarca la panorámica actual - dominante- de la investigación científica. A través de esta corriente se establece que el único conocimiento posible es aquel contrastado mediante el método científico como, por ejemplo, el trabajo experimental realizado en un laboratorio. Esta forma de entender se encuentra dentro de los enfoques cerrados que identificarán Everston y Green en (1989). 
Pero, si es así, ¿dónde queda el conocimiento de las ciencias sociales? Comprender el comportamiento del ser humano, las conductas y decisiones que le afectan en su día a día se escapa de las posibilidades de un laboratorio, puesto que el ser humano alberga conceptos, sentimientos y actitudes que van más allá de lo palpable y lo cuantificable. La tendencia es hacer todo medible y controlable para poder establecer patrones generalizables que se terminan presentando como modelos a seguir o aplicar, o incluso bajo el engañoso término de "buenas prácticas". Estos conocimientos establecidos como verdades absolutas tienen la limitación de que están condicionadas por el alcance que tiene el ser humano de acceder a la información y el conocimiento en el momento en el que realiza dicha investigación (Flick, 2018). Podemos decir, sin errar demasiado, que las fronteras del conocimiento del ser humano se amplían, y lo que hoy es considerado como una supuesta verdad incuestionable, dejaría de serlo y se vería cuestionada. Además, así entendido el conocimiento, supone, al mismo tiempo, la exclusión de otros conocimientos y formas de conocer que se entienden como no legítimos, no autorizados. Por tanto, para la comprensión del mundo y del ser humano (y de la educación) se debería tener en cuenta que el conocimiento no es neutral y no está al margen de factores políticos, sociales, económicos, etc., que inciden en el mismo (pensemos en la pandemia que estamos viviendo). El conocimiento que vamos teniendo de la realidad es una representación guiada interpretativamente del momento en el que se está viviendo y no una verdad absoluta válida y eterna. Hay que buscar una vía alternativa, y la tenemos: la investigación cualitativa (Denzin y Lincoln, 2018).

\subsubsection{Investigación cualitativa}

Desde las consideraciones anteriores, nos posicionamos en la investigación cualitativa por entender que con esta práctica la comprensión de la realidad educativa para su transformación es la adecuada. Cuando hablamos de investigación cualitativa hacemos referencia a

cualquier tipo de investigación que produce hallazgos a los que no sellega por medio de procedimientos estadísticos u otros medios de cuantificación. Puede tratarse sobre la vida de la gente, las experiencias vividas, los comportamientos, emociones y sentimientos, así como al funcionamiento organizacional, los movimientos sociales, los fenómenos culturales y la interacción entre las naciones. Algunos de los datos pueden cuantificarse, por ejemplo, con censos o información sobre los antecedentes de las personas u objetivos estudiados, pero el grueso del análisis es interpretativo. (Strauss y Corbin, 2002 en Toro y Parra, 2010, p.60)

Es por ello que se podría afirmar que, al menos, una de las finalidades que podemos considerar clave de la investigación cualitativa es "comprender e interpretar la realidad tal y como es entendida por los sujetos participantes en los contextos estudiados” (Rodríguez, Gil y García, 1999, pp. 259-260). Asumir esta forma de hacer investigación supone comprender las relaciones humanas y los fenómenos sociales que tienen lugar en un contexto educativo en cuestión, con el fin de interpretarlo y comprenderlo (Cruz, 2014). Esa comprensión se consigue a partir de la reflexión, del análisis y de la interpretación, situándonos en los contextos educativos y en los puntos de vistas de las personas pertenecientes a estos contextos. Asimismo, se trata de una investigación más humana, aportando una interpretación sobre una realidad con la intención de mejorarla o comprenderla (Contreras y Pérez de Lara, 2010). 
En consonancia con lo expuesto, un aspecto muy importante a la hora de llevar a cabo investigaciones cualitativas, es que se debe tener en cuenta la necesidad de ponerse a la escucha (Contreras y Pérez de Lara, 2010). Y no solo sentirse con la potestad de "dar voz" a aquellas personas a las que les hemos solicitado información acerca del ámbito que se investiga, ya que ponerse a la escucha "no es sólo escuchar sino también abrirse a la experiencia de escuchar. Porque ello nos exige descolocarnos, para hacer lugar al otro, a la otra, y la palabra que trae” (Molina, 2014 citado en Blanco y Sierra, 2017, p.7). En este ejercicio de ponerse a la escucha, se han de tener en cuenta algunos de los principios éticos como los propuestos por Vázquez y Angulo (2003, p. 21), los cuales serán tratados más adelante, y son: Negociación, Colaboración, Confidencialidad, Imparcialidad, Equidad y Compromiso con el conocimiento.

Asumimos, por tanto, que investigar en educación y sobre educación se precisa anclarnos en la investigación cualitativa, y ha sido precisamente esta en la que nos hemos situado en nuestros primeros pasos por el mundo de la investigación. A continuación, damos paso a narrar las experiencias vividas en nuestras primeras investigaciones a través de diferentes fases que incluyen aspectos tratados en los apartados anteriores.

\section{LA INCERTIDUMBRE DEL PRINCIPIANTE}

\section{1 ¿Qué queremos investigar? Llega la primera decisión}

Una vez que "finalizamos" la formación en investigación a través del máster realizado, llegó el momento de la verdad, el momento de poner a prueba todo lo aprendido y comenzar una investigación que nos pondría en contacto con la realidad de nuestro contexto. A lo largo de este apartado, expondremos desde nuestra experiencia los aspectos a destacar a la hora de enfrentarse a una investigación. Desde nuestro punto de vista, para llevar a cabo una investigación, sin duda la temática elegida para llevarla a cabo tiene que ser una que nos motive, preocupe o llene, ya que únicamente sintiendo la causa del hecho acontecido se puede empatizar y dar respuestas a las necesidades presentadas de manera más acertada. A pesar de ello, este punto que resulta tan transcendente y clave, en muchas ocasiones supone el primer hándicap al estar, dicha investigación, inmersa dentro del ámbito académico, y es que,

Los trabajos universitarios de investigación han estado sujetos a bastante crítica, por parte de la comunidad académica y por el público en general. El primer proyecto de investigación de un estudiante es, por lo común, una exigencia de la Universidad para satisfacer en parte los requisitos de un curso o para la obtención de un grado superior. La motivación inicial puede no ser el deseo de entregarse a la investigación, sino la necesidad práctica de satisfacer la exigencia. (Best, 1982, p. 36)

Sin embargo, resulta necesario tener en cuenta que esto no es siempre así y que en la mayoría de los casos se busca que, como alumnos y alumnas, tengamos la oportunidad de comenzar a investigar acerca de un ámbito de interés. En esta línea, poner de manifiesto algunas cuestiones que suelen ocurrir cuando se van a llevar a cabo las primeras investigaciones de la mano de un 
Trabajo Fin de Grado (TFG) o Trabajo Fin de Máster (TFM), y que pueden darse en posteriores prácticas investigadoras:

- Los alumnos y las alumnas nos centramos en elegir la temática que deseamos sin tener en cuenta otro requisito.

- Los alumnos y las alumnas elegimos, en primer lugar, a los docentes que ofertan dicha temática para asegurarnos una buena guía y tutorización, independientemente del asunto a tratar.

- Los alumnos y las alumnas coincidimos en temática y tutor o tutora, pero encontramos algún tipo de dificultad u obstáculo a la hora de desarrollar el tema deseado, ya que hay que tener en cuenta que dicha investigación cuenta con unas características concretas en cuanto a tiempo y forma por encontrarse inmersa en un marco académico y con unos plazos definidos.

\subsection{Plantear objetivos, ¿es tarea fácil?}

Cuando nos adentramos por primera vez en un proceso de investigación, es muy frecuente tender a querer abarcar el ámbito elegido desde una perspectiva muy amplia y ambiciosa, ya que existen muchas ganas de poner en práctica todo lo aprendido. A pesar de ello, no hay que olvidar que probablemente contamos con un tiempo concreto, algo que sin duda determinará en cierto modo el desarrollo de la investigación, siendo necesario establecer una pretensión del estudio acorde con nuestros conocimientos, así como concretar unos objetivos y unas cuestiones de investigación que den respuesta a las intenciones planteadas, siempre de manera coherente con el tiempo disponible y no planteando objetivos inabarcables, suponiendo todo esto un primer encuentro con la incertidumbre y las dudas.

No obstante, debemos tener en cuenta que, aunque a priori se planteen unos objetivos de investigación y una forma de hacer investigación, la persona encargada de investigar debe saber que este proceso se construye en la práctica y durante su desarrollo, por lo que se tomarán decisiones a lo largo de todo el camino, que quizás harán que algunos de esos objetivos se vean alterados, otros de los considerados se verán alcanzados y otros no, ofreciendo opciones de mejora que probablemente puedan desembocar en nuevas y futuras investigaciones.

En nuestro caso, para ambos el diseño inicial de la investigación fue cambiado por la casuística encontrada (temporalidad y características del contexto), generándonos un sentimiento de desconcierto y descontrol sobre el proceso de investigación. Por ello, consideramos oportuno mencionar que no existen unas directrices pautadas de los procesos de investigación, sino que se trata de un proceso flexible y cambiante que se va conformando y modificando durante la investigación, particularidades que son propias de la investigación cualitativa. Se van perdiendo los miedos.

\subsection{Navegar en investigaciones previas}

Como es lógico, y teniendo en cuenta que una investigación cuenta con diferentes etapas y momentos que generan distintas necesidades, la persona que investiga se sentirá más o menos a gusto en cada una de estas etapas. En nuestro caso, sabemos que uno de los aspectos que más influye en la elaboración de la investigación es el marco teórico, siendo necesario tener un bagaje previo de las investigaciones realizadas en torno a la temática a tratar y también sobre el estado de la 
cuestión. Es decir, resulta fundamental poder argumentar y facilitar a las personas receptoras un marco teórico que avale, respalde y justifique el motivo de realizar esa investigación y no otra, dotando de importancia y coherencia al proceso indagatorio. De igual forma, resulta un arduo proceso de aprendizaje y reflexión, comprender que un marco teórico no es fruto de un resumen de todas las lecturas realizadas, sino que implica reflexionar sobre las mismas para así elaborar un discurso propio y argumentado, ya que en muchas ocasiones una investigación surge de otras predecesoras.

Esta última idea nos lleva, además, a un aspecto muy importante y fundamental del proceso como es la búsqueda de bibliografía relacionada con la temática que estemos trabajando. Desde nuestro proceso, dicha bibliografía debe cumplir con ciertas características como:

- Ser variada, no recurriendo en muchas ocasiones a la misma fuente de información.

- Estar actualizada, ya que probablemente al hablar de investigación, los resultados y las conclusiones obtenidos hace quince años sobre cualquier temática hoy en día no serán los mismos, a no ser que la fuente de información primaria sea requerida o determinante a la hora de desarrollar la investigación.

- Contar con diferentes fuentes (libros, artículos, revistas, entrevistas) que nos aporten una visión amplia de la temática, siempre que las mismas cumplan criterios de rigor y credibilidad.

A ello se le une una cuestión que es aún más importante, si cabe, y es que se ha de destacar la relevancia de formarnos para citar correctamente las fuentes utilizadas, tanto en el cuerpo de texto (desarrollo) como en el apartado específico de las referencias bibliográficas. Hemos aprendido sin duda la importancia de respetar la autoría de los documentos, valorando el trabajo realizado por otros y otras profesionales.

\subsection{Estrategias metodológicas para recogida de información}

Una vez que tengamos los objetivos y las cuestiones de investigación a las que se les desea dar respuesta, es conveniente pararse a reflexionar acerca de cuáles serán aquellas estrategias de recogida de información que mejor se ajusten a la investigación en cuestión, así como diseñar instrumentos para poder llevar a la práctica dichas estrategias, intentando, a nuestro parecer desde la propia experiencia vivida que estos instrumentos en todo momento:

- No pretendan recoger de manera indirecta la información que nos interese para nuestra investigación.

- Evitar sesgos (género, social, cultural, étnica, etc.).

- Coherencia con la metodología elegida (cualitativa).

Concretamente, nosotros tuvimos la oportunidad de iniciarnos en el empleo de diferentes estrategias que se ajustaban a las necesidades de la investigación, dato que hay que tener en cuenta, ya que en algunas ocasiones queremos llevar a cabo diversas estrategias por tener la oportunidad de adentrarnos en todas, pero que no se ajustan a las necesidades requeridas por parte de la investigación, el contexto y el tipo de personas participantes, por una parte, pero también, y por otra, por coherencia epistemológica con la forma de hacer investigación (cualitativa). Durante 
dicho proceso, lo cierto es que obtuvimos sentimientos encontrados, dado que hay que tener en cuenta que en muchas ocasiones la realidad vivida altera los pasos teóricos que conforman el empleo de una determinada estrategia, resultando necesario ajustar nuestra forma de proceder o actuar. Todas estas experiencias serán narradas en los siguientes apartados.

\section{La Observación:}

Cuando comenzamos las investigaciones, sin duda un cuaderno fue nuestro gran aliado y es que desde el minuto uno en el que accedimos al contexto correspondiente o simplemente desde el momento en el que comenzamos a indagar y realizar una revisión bibliográfica del tema a tratar, fue interesante, y muy necesario, comenzar a anotar todo aquello que nos rodeaba o que llamara nuestra atención, porque cualquier dato podía ayudarnos a comprender mejor aquello con lo que trabajábamos. Además, hizo que pudiéramos ir en busca de ciertos datos que por alguna razón hubiéramos olvidado, descubrir que algunos aspectos concretos se repetían con frecuencia entre otros muchos, o abrirnos nuevas vías para ir focalizando la problemática de nuestro estudio.

\section{Las Entrevistas:}

La entrevista, para nosotros, es la estrategia por excelencia, ya que nos permite compartir espacio y tiempo con las personas participantes de una manera cercana y empática, lo que favorece la escucha, conociendo de manera detallada las percepciones y opiniones de las personas participantes (Wood y Smith, 2018). A pesar de ello, a la hora de ponerla en práctica hemos podido comprobar que en ocasiones resulta un poco complicado crear un espacio distendido entre la persona investigadora y la persona entrevistada, teniendo en cuenta que se le van a hacer una serie de preguntas que no sabrá cuáles son y que serán, además grabadas (bajo su consentimiento). ¿Qué queremos decir con esto? Pues que la verdadera entrevista dista de poder hacer de ella la recreación de un encuentro entre dos personas de manera natural que, tras encontrarse en una cafetería, comparten temáticas y preocupaciones en común. De ahí que nuestra actitud, tono o cercanía serán nuestros únicos aliados para conseguir realmente un espacio para la escucha atenta de las personas entrevistadas y lograr con ello acercarnos de manera comprensiva a la realidad objeto de estudio; aportaciones que conecten con nuestras cuestiones de investigación. Nos dimos cuenta de que la realidad es la que manda (en gran parte). En relación con este aspecto, resulta muy importante destacar que la persona investigadora nunca puede obviar el compromiso ético con las personas, proporcionando un ambiente cómodo, empático, donde las personas participantes puedan expresar su visión sin presión. Por ello, en ningún momento aportamos nuestra opinión personal en las respuestas que se fueron recibiendo (no estábamos para juzgar).

Algo muy importante antes de llevar a cabo la entrevista fue formular un buen guion, o al menos intentamos que lo fuera, ya que las preguntas realizadas serán fundamentales para conseguir indagar acerca de la temática deseada, agradeciendo siempre la colaboración de las personas, abriendo una puerta para que ellas pudieran expresar las ideas que desearan. Asimismo, también es importante dejar un guion abierto a posibles preguntas emergentes (algo exigido para una investigación que le interesa las significaciones y los modos con los que las personas representan la realidad), ya que durante la entrevista pueden salir algunas temáticas no contempladas con anterioridad. Lo cierto es que nuestra experiencia a la hora de realizar entrevistas 
fue muy buena, puesto que las personas entrevistadas se mostraron interesadas en el ámbito investigado y sentían como suya la investigación. Gracias a ello la información recabada resultó interesante para los propósitos que se tenían.

\section{Grupo focal:}

La realización de un grupo focal puede resultar algo complejo por requerir citar a varias personas en un mismo lugar y hora para que puedan conversar acerca del tema que estemos tratando (Kambeleris y Dimitriadis, 2013). Sin embargo, a la vez nos parece muy recomendable porque ofrece una visión muy completa de la temática de estudio por poder incluir diferentes visiones desde la construcción del discurso entre las personas participantes. Concretamente la experiencia vivida fue muy buena, y se llevó a cabo con alumnos y alumnas de $2^{\circ}$ de Bachillerato, los cuales tenían en común la participación en un mismo programa de convivencia desarrollado en el centro. De tal forma que la experiencia de este vínculo y el participar juntos y no de manera individual, propició una conversación muy fluida y sobre todo que se mostraran bastante relajados. En este caso concreto, la realización de un grupo focal se vio enriquecido por la aportación de las pruebas documentales, tratadas a continuación.

\section{Pruebas documentales:}

Las posibles fotografías o los vídeos realizados, con su correspondiente autorización, pueden ayudar mucho a la hora de extraer datos relevantes, dado que no podemos estar atentos a toda la información, movimientos y acontecimientos que se produzcan en el contexto del trabajo de campo, por lo que dichas fotografías y vídeos nos permitirán volver a vivir ciertas actividades, situaciones, acciones o conversaciones proporcionándonos la oportunidad de ver algunos aspectos que durante la realización de los hechos no tuvimos en cuenta, o teniéndolos, profundizar en los mismos.

A lo largo de una de las experiencias vividas se pudo en valor la importancia de estas y es que en este caso las fotografías y los videos, además de usarse las realizadas en el momento en el que se estaba llevando a cabo la investigación, también se emplearon documentos gráficos con los que se contaba desde hacía varios años atrás. Estas últimas, procedentes de una investigación que precedía a la realizada para la elaboración del TFM, sirvieron para comparar una misma situación a lo largo del tiempo y poder ver cómo esta había cambiado o no, ya que en este caso la investigación consistía en conocer el impacto que había tenido la implementación de un proyecto alumno ayudante en un instituto de educación secundaria de la provincia de Cádiz.

\section{Conversaciones informales:}

Esta estrategia, a pesar de tener atribuida la coletilla de informal, no dota por ello de menor importancia, ya que puede suponer una fuente de información que de otro modo no resultaría posible disponer. Esta peculiaridad de la herramienta en cuestión se debe a que al no estar planificada fluye de manera más natural, colocando a la persona participante en una situación más relajada y con más disposición a compartir, al menos es lo que pudimos apreciar con nuestras investigaciones. 
Dado que no es un encuentro planificado, como es el caso de la entrevista, es muy oportuno contar siempre con una libreta o algún soporte donde anotar diferentes comentarios o datos que vayan surgiendo. No obstante, durante el trabajo de campo éramos conscientes de que la información lograda mediante conversaciones informales era registrada fuera del escenario o campo consensuado, por ello, la información recabada no podría ser incluida en la investigación, sin previo consenso. De manera resumida, las conversaciones informales suponen un espacio de diálogo más relajado y natural, no premeditado, donde las personas informantes pueden aportar datos desde un punto de vista más personal y natural de gran valor para la investigación.

\subsection{Personas participantes, ¿requiere compromisos especiales?}

Un aspecto crucial a la hora de llevar a cabo una investigación, y sobre todo una investigación con metodología de corte cualitativo, es que es necesario establecer unos principios éticos. Esto es así porque se tiene la oportunidad de estar en contacto directo con las personas participantes, las cuales nos ofrecerán información valiosa del ámbito que se pretende indagar. Y es que no es hasta el momento en el que nos adentramos realmente en una investigación, cuando conoces los muchos aspectos necesarios que se hemos de tener en cuenta, y que son los ya mencionados con anterioridad. La persona que lleva a cabo la investigación nunca debe desprenderse de su versión más humana (porque tampoco puede ser de otro modo por coherencia con la forma de investigación elegida), teniendo en cuenta que los datos en este tipo de investigación provienen de un contexto social, formado por personas que sienten los fenómenos que se dan en el mismo, cargados de significados. Por ello, resulta necesario poner en bandeja una serie de valores, no solo de investigación, sino sobre todo humanos que, ante todo, protejan la integridad y el bienestar de las personas que contribuyen en la investigación, así como la relación entre las mismas. A continuación, se exponen algunas anotaciones relacionadas en relación a dichos principios éticos en nuestras investigaciones:

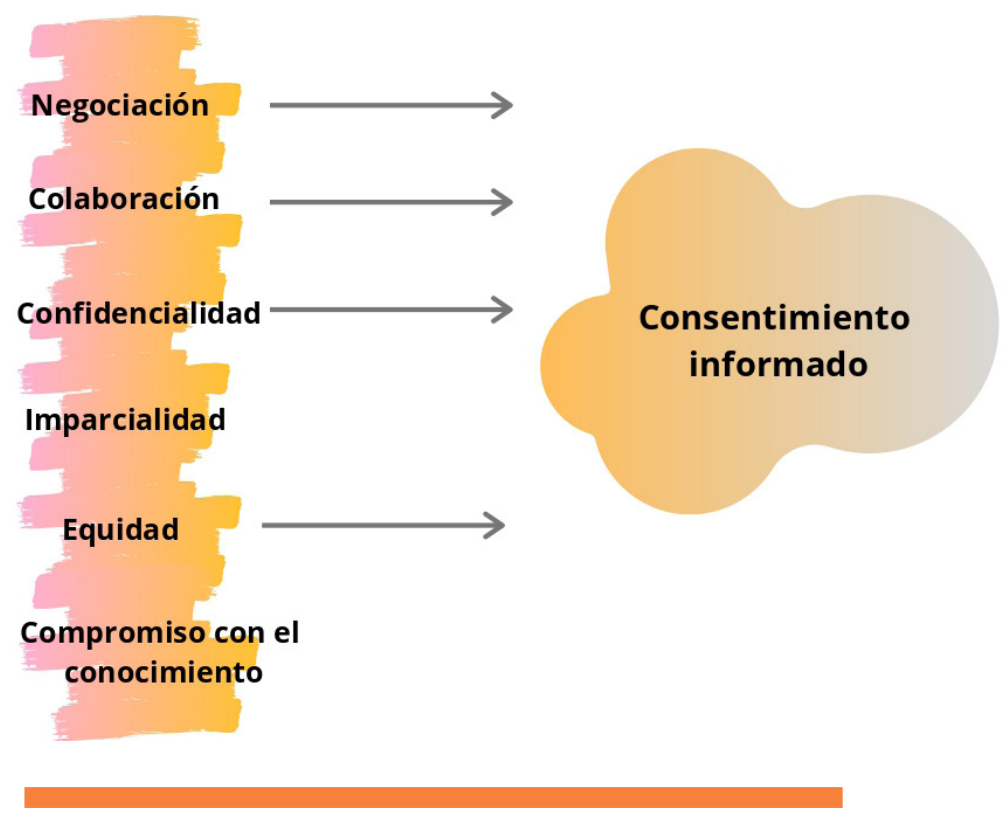

Figura 1. Principios éticos. Fuente. Elaboración propia 


\section{E S T U D I A N T E S}

Como se puede ver en la figura 1, existen algunos principios éticos a tener en cuenta que son considerados en un documento denominado consentimiento informado. Dicho documento refleja aspectos como en qué consiste la investigación que se va a realizar, por qué es importante el testimonio de la persona o las personas a las que se les va a entrevistar, pero, sobre todo, cumple una función muy importante que es hacer constar que la persona participante autoriza a trabajar con la información recabada, conociendo que será algo totalmente confidencial. A continuación, se puede ver un ejemplo de este documento:

\section{UUCA tocisted}

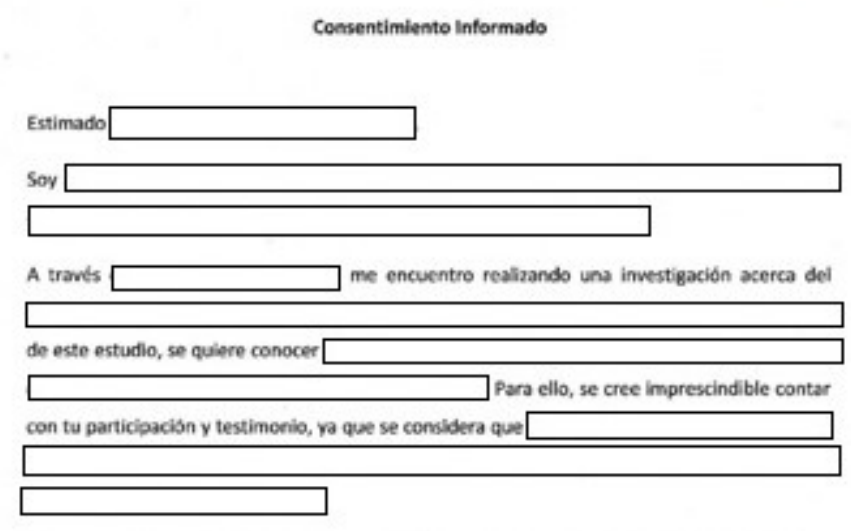

Tu participación en la realización del grupo focal es totalmente voluntaria, teniendo el derecho de retirar el consentimiento para la particlasción en cualquier momento. Del mismo modo, debe ser informado o informada de que la conversación será grabada pudiendo acceder a la transcripción de dicho documento por si desea modificar algunos datos que haya ofrecido, asi como a acceder a los resultados finales de la investigacion.

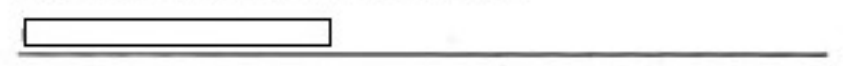

AUTORZZACIÓN

He leido el procedimiento descrito arriba. La imvestigadora me ha explicado el estudio y ha contestado a mis preguntas. Voluntariamente doy mi consentimiento para particioar en la

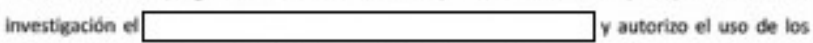
datos que se extraigan de las entrevistas en las que participe para este trabajo académico, así como para la difusión cientifica de resultados. He recibido copia de este procedimienta.

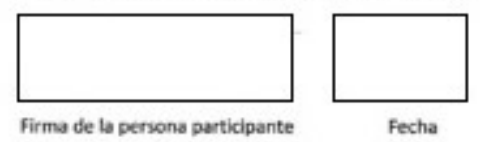

Firma de la persona participante

Fecha

Figura 2. Consentimiento informado. Fuente. Elaboración propia para ser aplicado en una de las investigaciones realizadas

Pasamos de la teoría a esos principios llevados a la práctica, esto es, a nuestras experiencias investigadoras. ¿Qué podemos contar?

Negociación: tiene que estar presente a lo largo de toda la investigación y no solo al inicio de la misma. Por ejemplo, a la hora de determinar el tema a tratar o para confirmar las perso- 
nas que participarán en la investigación, entre otros, ya que a lo largo de todo el proceso se irán realizando avances que también deberán ser negociados como la manera en la que será registrada la información facilitada o incluso la información plasmada en el informe final. Lo que nos lleva a amparar la idea de respetar los límites de la información, además de brindarles a las personas participantes la oportunidad de realizar las preguntas que consideren oportunas.

El hecho de respetar los límites de la información se ve reflejado en que, por ejemplo, desde nuestra experiencia, a la hora de llevar a cabo la entrevista, contamos con la participación de personas participantes que no querían responder a algunas preguntas y preferían reservarse alguna información. Por ello, como personas investigadoras tuvimos que respetar la decisión y que la personas contase lo que considerasen oportuno. Sí pudimos experimentar que, una vez apagada la grabadora, estas personas se relajaban y comenzaban a contar aspectos tratados a lo largo de la entrevista pero, en este caso, con un mayor trasfondo. Sin embargo, resulta imprescindible mantenerse fiel a los compromisos éticos y no revelar información que la persona informante no desee. Esta información suponía confidencialidad, puesto que las personas informantes no querían que apareciese en los datos recogidos de la investigación.

Colaboración: será esencial también poner en conocimiento de las personas participantes la posibilidad tanto de formar parte de la investigación como de dejar de hacerlo en el momento que crean oportuno. Además, en cuanto a la información revelada por parte de las personas participantes se ha de destacar y dejar claro que la participación de las mismas en el estudio es totalmente voluntaria, por ello los y las participantes aportarán la información que consideren oportuna, profundizando en mayor o menor medida en los aspectos tratados.

Confidencialidad: este principio resulta uno de los más importantes, ya que en algunas ocasiones es posible que la información que estemos solicitando sea de carácter delicado, pudiendo poner en un compromiso a la persona que ofrece la información. Por ello, en todo momento nos aseguramos tanto del anonimato de las personas como de la confidencialidad de la información dada, no relacionando en ningún momento esta con la persona que la aporta. Además, siempre se debería incluir la posibilidad de que la información recabada sea mostrada al participante otorgándole la oportunidad de modificar si lo desea, ya que puede que la persona finalmente no se vea reflejada en las opiniones vertidas o no fuera eso precisamente lo que desea dar a conocer.

Imparcialidad: En cuanto a este principio, hemos de señalar que repercute principalmente en dos momentos de la investigación. Por un lado, en el análisis de los datos recogidos, y, por otro, a la hora de recabar la información porque la persona investigadora, con sus gestos o apoyos verbales, inducir a ciertas respuestas que le interese escuchar para su investigación, obteniéndose así una información sesgada de la realidad. Este es un aspecto que como personas investigadoras noveles hemos tenido presente a lo largo del proceso de recogida de información, mostrándonos imparciales ante los datos recogidos y apoyando a las personas participantes sin interceder u opinar acerca de su planteamiento o idea. De ahí la necesidad de incluir en nuestra investigación el concepto de "extrañamiento", a través del cual como investigador o investigadora se ve o interpreta todo el contexto de trabajo desde una perspectiva distinta, "nueva”, la que es propia de las personas participantes; los lugares y las personas conocidas verlos con otra mirada como si fuese nuevo en ese lugar y no se supiera nada acerca del mismo ni de las relaciones que lo rigen. 
Equidad: Este aspecto es esencial, ya que, en muchas ocasiones desde el papel investigador nos creemos con el poder de enjuiciar lo encontrado o de manejar la situación encontrada, dejando a un lado las experiencias y vivencias de las personas participantes. Por ello, a través de este principio se asegura el buen trato de las mismas, ofreciéndoles la oportunidad de modificar aquello que han aportado cuando estimen oportuno. Así mismo, este principio implica dar la oportunidad a todas las personas que forman parte del contexto investigado como informantes si asílo desean.

Compromiso con el conocimiento: Este último principio hace referencia a nuestro compromiso como personas investigadoras de aportar conocimiento siempre y cuando se investigue hasta donde sea posible, hasta donde las circunstancias y las personas participantes nos lo posibiliten y, además, teniendo siempre presente el cumplimiento del resto de principios éticos mencionados con anterioridad.

\section{6 ¿Qué narran los datos? Toca analizar}

Uno de los puntos que se piensa más dificultoso a la hora de llevar a cabo una investigación es el momento en el que se comienza a analizar toda la información que se ha ido recabando a lo largo de todo el proceso (Fernández y Del Valle, 2017). En este sentido, aunque se supone que esta dificultad es algo que puede llegar a ocurrir, con independencia de los años que lleve el investigador o la investigadora realizando pesquisas, se considera que esta tendencia aumenta aún más si se trata de la realización de las primeras investigaciones (Vázquez, 2011), ya que una de las principales ideas que viene a la cabeza es "quién soy yo para interpretar esto”, "quién soy yo para decir que esto es de este modo". Suponemos que este "miedo" va desapareciendo poco a poco a medida que nos vamos involucrando en la temática, pero, sobre todo, a medida que vamos aprendiendo nuevas formas para argumentar las conclusiones, organizar y estructurar la información conforme a las categorías que se han ido configurando con el análisis, y a medida que vas aprendiendo a distinguir tu "yo" investigador de tu "yo" posicionado ante una realidad, consiguiendo el equilibrio entre ambos, o al menos intentándolo en todo momento.

Una vez que se recogen los datos el dilema que surge es el siguiente, ¿cómo interpretar los datos desprendiéndome de mi propia visión?, ¿estoy sacando conclusiones creíbles o tienen el sesgo de mi postura personal? Ir más allá de los números y trabajar con las vivencias y los sentimientos de personas implica romper con la cualidad cuadriculada de los números para pasar a lo social y, en este sentido, resulta esencial interpretar los datos de una manera imparcial y extraer las conclusiones y no persiguiendo resultados que interesen al investigador o la investigadora. La interpretación la hacemos poniéndonos en el punto de vista de las personas participantes, suponiendo este un gran esfuerzo, ya que no estamos acostumbrados a este tipo de tareas. A pesar de ello, y de trabajar con los datos de manera rigurosa y ética, cabe decir que, de manera inevitable, la percepción de la persona investigadora, su experiencia y sus vivencias, intercederán en la interpretación que esta haga de la información extraída; no podemos desprendernos de nuestra subjetividad, y de la intersubjetividad construida a lo largo del proceso de investigación. Por ello, se habla de miradas interpretativas en tanto que persiguen comprender las interacciones que se dan en un contexto determinado (Denzin y Lincoln, 2018), así como los fenómenos que interceden en esta realidad, pero sin el propósito de generar teorías generalizables aplicables a otros contextos en su totalidad como puede ocurrir con las metodologías experimentales. 
En esta línea, otra dificultad que se nos presentó fue la de establecer las categorías para el análisis de la información, procedimiento relativo a la fase analítica de la metodología cualitativa para el tratamiento de datos. Para ambos, y para muchos y muchas de nuestros compañeros y nuestras compañeras, este es uno de esos conceptos más complejos de llevar de la teoría a la práctica. Es decir: ¿cómo establecer núcleos de interés o temáticos para el análisis de los datos?, ¿cuáles son las temáticas que más se repiten a lo largo de la información? Sin duda, es una decisión crucial e importante para la obtención de los resultados, pues una vez establecidas las categorías, serán las que nos ayuden a estructurar con sentido, coherencia y comprensividad el análisis. Esto requiere de mucha revisión de la información recabada y control del tema (vivir los datos, hacerlos nuestros) para poder conocer cuáles son aquellos aspectos que toman más protagonismo a lo largo de la información y en qué ámbitos temáticos pueden recogerse.

\subsection{El sentido de la investigación, ¿cómo concluimos?}

Este apartado de la investigación, a pesar de ser uno de los más importantes y determinantes, fue uno de los que más nos costó, porque sentíamos que ya se había dado toda la información necesaria que teníamos a lo largo de todo el documento del trabajo de investigación, sintiendo no poder ofrecer más información a la persona lectora. Sin embargo, tal y como se apunta, es esencial y pueden existir tantos tipos de informes como investigadores e investigadoras realizan estudios.

Además, concluir en sí mismo no es fácil tampoco, porque no siempre se tiene claro cuándo y cómo hacerlo. Al margen de la dificultad mencionada, consideramos que las conclusiones son necesarias porque dan respuesta directa a los objetivos planteados inicialmente. Asimismo, ponemos en valor aportar un feedback a las personas informantes en cumplimiento al principio de negociación; también porque, si la realización de una investigación solo tiene el fin de emitir un artículo o realizar un TFM para obtener una calificación, ¿qué sentido tiene investigar? Para nosotros, después de este proceso, nos resulta crucial aportar y compartir los resultados con las personas participantes con la intención de contribuir a esa mejora de las prácticas educativas en el contexto en cuestión, pues qué es sino la investigación educativa y la investigación para la educación (la primera al servicio de la segunda).

\section{4. ¿QUÉ HEMOS APRENDIDO HASTA AHORA? ¿CÓMO VEMOS EL FUTURO?}

Para finalizar con este trabajo realizado desde sujetos neófitos, se considera oportuno poner de manifiesto las impresiones que como personas inexpertas en el ámbito de la investigación nos hemos llevado. No desde el juicio de la verdad, pero sí desde nuestra experiencia personal.

En este sentido, consideramos importante poner en relevancia el papel de la investigación como recurso que permite al personal inmerso en el ámbito educativo conocer la casuística presente en los contextos educativos, así como descubrir e implementar nuevos conocimientos, estrategias y metodologías que mejoren la práctica educativa.

Pero, ¿es esto siempre así? Desde lo aprendido y experimentado, hemos podido ver cómo, aun siendo los maestros y las maestras de primaria e infantil las personas que más en contacto se encuentran con los niños y las niñas en el ámbito académico, es el personal vinculado al 
ámbito universitario quien más investiga, a través de la experiencia propia y de los contenidos trabajados a lo largo de diferentes asignaturas. ¿Por qué esto es así? Desde nuestra experiencia, hemos podido comprobar cómo en muchas ocasiones el profesorado de los centros educativos de educación infantil y primaria alega que este tipo de investigaciones no son posibles debido al volumen de trabajo con el que se encuentran en el día a día en sus aulas, y todas las diligencias propias del ámbito, sobre todo, con relación a la burocracia exigida a nivel administrativo. Sin embargo, creemos que en muchas otras ocasiones el profesorado realiza investigaciones en el aula sin apenas darse cuenta, con el simple hecho de observar una determinada conducta en algún alumno o alguna alumna, anotar sus avances, preguntarle qué le pasa, informarse acerca de cómo puede ayudarlo y finalmente concluir con algunas respuestas al respecto. Asimismo, otro motivo por el que los maestros y las maestras no investigan ni constan en publicaciones del ámbito educativo, según hemos podido experimentar, es la ausencia de formación en el planteamiento de los grados de Educación Primaria e Infantil en cuanto al ámbito de la investigación. Por ello, el profesorado no cuenta con las herramientas y los recursos que les permitan formalizar los procesos de investigación y poder escribir unos resultados que se emitan a la comunidad educativa. Finalmente, en cuanto a este colectivo mencionar que la ausencia de formación en los planteamientos del grado, en lo que se refiere a la investigación, tal y como hemos podido comprobar desde nuestra experiencia personal, hace que no exista conciencia o interés sobre la importancia de la investigación para la práctica docente y educativa.

Dejando a un lado las esferas de infantil y primaria, y dando paso al ámbito universitario, nos gustaría poner de manifiesto el sabor agridulce que ha impregnado nuestros pensamientos a lo largo de esta experiencia. Siendo delicada la temática y sin juzgar la voluntad de la calidad docente que nos ha rodeado durante esta etapa, cabe preguntar: ¿determinan las exigencias del ámbito universitario el tipo de investigaciones que se llevan a cabo?, ¿por qué investigan más en el ámbito universitario?, ¿supone investigar una motivación intrínseca o una exigencia externa del ámbito laboral? Son algunas preguntas que navegan por nuestra mente, siempre hablando desde la estructura y el planteamiento de admisión para acceder al mundo laboral de la universidad. Desde nuestro punto de vista, emitir artículos en el ámbito universitario se convierte en una exigencia y hándicap para el profesorado, que se ve empujado y sometido a una presión que les exige escribir. Esto no implica que estas investigaciones realizadas para publicar dichos artículos no sean de calidad, pero, ¿la temática investigada surge de un interés o duda personal o viene determinada por los intereses globales o "de moda"? Lo que se pretende transmitir con esta idea última es la importancia y el peso que tiene para la comunidad universitaria la publicación en revistas de impacto, ya que cuentan con un mayor baremo a la hora de evaluar su currículum.

Por último y para concluir, desde nuestra modesta experiencia y opinión, alegamos enaltecer el papel de la investigación como estrategia que ayuda a la comunidad educativa a comprender los fenómenos que en este ámbito intervienen, así como mejorar en cuanto a metodologías y conocimientos para hacer cada día una mejor educación, más humana, equitativa y justa. Asimismo, alabar el papel de la comunidad universitaria en la realización en investigaciones, no desmereciendo su trabajo por la situación planteada anteriormente, sino como personas, y trabajadoras, maestros y maestras, que se ven perjudicados por esta situación actual del sistema universitario y que, en ocasiones, desvirtúa el papel de la investigación en educación. Por último, vemos la necesidad de establecer una conexión entre la universidad y la escuela que persiga tra- 
bajar conjuntamente por un mismo fin, huyendo de las jerarquías y figuras expertas. Han sido todos estos motivos los que nos ha llevado a desembarcar en el doctorado.

\section{REFERENCIAS}

Best, J.W. (1982). Como investigar en educación. Morata.

Contreras, J. y Pérez de Lara, N. (2010). La experiencia y la investigación educativa. En J. Contreras y N. Pérez de Lara. (Ed.), Investigar en la experiencia educativa (pp. 20-92). Ediciones Morata, S.L.

Cruz, A. (2014). Importancia de la investigación educativa. Consejo de Transformación Educativa. Recuperado de: https://cutt.ly/pgw6XiV

Denzin, N., Lincoln, Y. (Eds.). (2018). The SAGE handbook of qualitative research (5th ed.).Sage. https://www.qualitative-research.net/index.php/fqs/article/view/14

Domingo, A. (2020). Profesorado reflexivo e investigador. Propuestas y experiencias formativas. Narcea.

Everston, C. M. y Green, J.L. (1989). La observación como indagación y método. En M.C. Wittrock (Ed.), La investigación como enseñanza. Métodos cualitativos y de observación. Vol. II (pp.303-430). Paidos/ MEC.

Fernández, M.A. y Del Valle, J. (2017). Cómo iniciarse en la investigación académica. Una guía práctica. Fondo Editorial.

Flick, U. (2018). The Concepts of Qualitative Data: Challenges in Neoliberal Times for Qualitative Inquiry. SAGE journals, 25 (8), 713-720. https://doi.org/10.1177/1077800418809132

Kamberelis, G. y Dimitriadis, G. (2013). Grupos focales. En N. K. Denzin y Y. S. Lincoln. (Ed.), Métodos de recolección y análisis de datos. Vol.IV (pp. 495-532). Editorial Gedisa, S.A.

Real Academia Española (2019). Diccionario de la Lengua Española. Edición de Tricentenario Recuperado de: https://bit.ly/35iLR7g

Rodríguez, G; Gil, J; García, E. (1999). Metodología de la investigación cualitativa. Ediciones Aljibe.

Sierra. E. y Blanco, N. (2017). El aprendizaje de la escucha en la investigación educativa. Qualitative Research in Education, 6(3), 303-326. doi:10.1783/qre.2017.2783

Tallaferro, D. (2006). La formación para la práctica reflexiva en las prácticas profesionales docentes. Educere, La revista venelozana de educación, 10(33), 269-273.

Tejedor, F.J. (2004). Investigación educativa: ¿Hacia dónde vamos? En L. Buendía, D. Gónzález, T. Pozo. (Coords.), Temas fundamentales de la investigación educativa (pp.63-104). La Muralla, S.A.

Toro, I. y Parra, R. (2010). Fundamentos epistemológicos de la investigación y la metodología de la investigación Cualitativa/cuantitativa. Fondo Editorial Universidad EAFIT.

Vázquez, R. y Angulo, F. (2003). Introducción a los estudios de caso. Los primeros contactos con la investigación etnográfica. Ediciones Aljibe.

Vázquez, R. (2011). Investigar con estudio de caso la dirección escolar. Ediciones Aljibe.

Wood, P. y Smith, J. (2018). Investigar en educación. Conceptos básicosy metodología para desarrollar proyectos 